\title{
Correction to: A Fully Pexiderized Variant of d'Alembert's Functional Equations on Monoids
}

Bruce Ebanks

\section{Correction to: Results Math https://doi.org/10.1007/s00025-020-01335-9}

Some errors in the above-named article need to be corrected. The most serious error occurs in Lemma 4.1, where some solutions were omitted. The mistake comes from the claim in the proof that $c=1 / 2$ (near the bottom of p.7), which is false. In fact after renaming $c$ as $c / a$ we get the following for part (i) of Lemma 4.1: For $\chi \circ \sigma \neq \chi$,

$$
\begin{aligned}
& f=\theta+b c(\chi+\chi \circ \sigma)+\frac{a b}{2}(\chi-\chi \circ \sigma), \\
& g=-\theta-b c(\chi+\chi \circ \sigma)+\frac{a b}{2}(\chi-\chi \circ \sigma), \\
& h=b(\chi-\chi \circ \sigma), \quad \text { and } \quad k=\frac{a}{2}(\chi+\chi \circ \sigma)+c(\chi-\chi \circ \sigma) .
\end{aligned}
$$

It can be checked that functions of such forms satisfy all the conditions of Lemma 4.1.

Also the reader should take $\mathbb{K}=\mathbb{C}$ throughout the paper, so $f_{o}$ is central in Lemma 4.4 and Theorem 4.6.

In Lemma 4.3, solution family (i) should state "For $\chi \neq \chi \circ \sigma$," since it arose under this condition in the proof. (In fact if $\chi=\chi \circ \sigma$ then the forms in (i) are contained in (ii).)

The original article can be found online at https://doi.org/10.1007/s00025-020-01335-9. 
The statement of Theorem 4.6 is correct, but part of the proof must be changed due to the mistake in Lemma 4.1. In the case $h \neq 0, k \neq 0, k(e) \neq 0$, and $h(e)=0$, solution (i) of Lemma 4.1 is solution (c) of Theorem 4.6 with $a_{1}=b c=-b_{1}, a_{2}=b_{2}=a b / 2, c_{1}=0, c_{2}=b, d_{1}=a / 2$, and $d_{2}=c$. It should also be noted that part (e) of Theorem 4.6 can be omitted, since it is contained in part (d) with $c_{2}=d_{2}=0$ (and a change of notation) because $\chi \in \mathcal{N}(S, \sigma)$ when $\chi=\chi \circ \sigma$.

There are small misprints on page 7 , where $(1 / 2) f_{o}=f-f \circ \sigma$ should be $2 f_{o}=f-f \circ \sigma$, and on page 11 where the displayed equation three lines above Case 2 should end " $=-(a / 4 b)\left[A^{2}(x y) \chi(x y)-A^{2}(\sigma(y) x) \chi(\sigma(y) x)\right] "$ instead of as printed.

The final remark is that in [1] Wilson studied the functional equation

$$
f(x+y)+g(x-y)=h(x) k(y),
$$

which encompasses both d'Alembert equations. Our principal functional equation is a variant of this for monoids.

\section{References}

[1] Wilson, W.H.: Two general functional equations. Bull. Am. Math. Soc. 31, 330334 (1925)

Bruce Ebanks

Department of Mathematics

University of Louisville

Louisville KY40292

USA

e-mail: ebanks1950@gmail.com

Publisher's Note Springer Nature remains neutral with regard to jurisdictional claims in published maps and institutional affiliations. 